\title{
Comparison of predictive value of NT- proBNP, sST2 and MMPs in heart failure patients with different ejection fractions
}

\author{
Wei Pan ${ }^{1,2+}$, Donghui Yang ${ }^{1,3+}$, Peng $\mathrm{Yu}^{4 \dagger}$ and Huizhen $\mathrm{Yu}^{1,4^{*}}$ (D)
}

\begin{abstract}
Background: This study sought to compare the predictive value of NT-proBNP, SST2 and MMPs in HF with different ejection fractions from a population in southern China.

Methods: A cross-sectional study was conducted on $113 \mathrm{HF}$ patients admitted to Fujian Provincial Hospital from December 2016 to March 2018. The patients were divided into three subgroups: 60 cases in HFpEF group (LVEF $\geq 50 \%)$, 28 cases in HFmrEF group (41\% $\leq$ LVEF $\leq 49 \%)$ and 25 cases in HFrEF group (LVEF $\leq 40 \%)$. ELISA method was applied to detect the concentrations of sST2, MMP-2 and MMP-9. Electrochemical luminescence immunoassay was applied to detect the concentration of plasma NT-proBNP. Univariate and multivariate Cox and logistic regression models were used to analyze the diagnostic significance of these plasma biomarkers in HF patients. Kaplan-Meier survival curves were used to assess the prognostic value of SST2 in the incidence of long-term adverse events during study.
\end{abstract}

Results: This study showed that plasma sST2 levels in HFrEF or HFmrEF patients were significantly higher than in HFpEF patients. Plasma levels of MMP-2 and MMP-9 in HFrEF patients were apparently higher than in HFpEF or HFmrEF patients. For the diagnosis of HFpEF, the AUC of NT-proBNP was higher than that of sST2, MMP-2 and MMP-9, which were $0.881,0.717,0.705$ and 0.597 , respectively. For the diagnosis of HFmrEF, the AUC of plasma sST2 was higher than that of MMP-2, MMP-9 and NT-proBNP, which were $0.799,0.678,0.676$ and 0.793 , respectively. For the diagnosis of HFrEF, the AUC of plasma NT-proBNP, SST2, MMP-2, and MMP-9 were 0.945, 0.820, 0.814, and 0.774 respectively. Spearman correlation analysis showed that plasma SST2 levels were significantly correlated with plasma MMP-2, MMP-9 and NT-proBNP levels. Further logistic regression analysis showed that except MMP-9, the biomarkers sST2 $(\mathrm{OR}=1.960)$, MMP-2 $(\mathrm{OR}=0.805)$ and NT-proBNP $(\mathrm{OR}=0.002)$ were all independent risk factors for patients with heart failure. Survival analysis results suggested that for patients with HFmrEF, a higher level of plasma sST2 $(\geq 0.332 \mathrm{ng} / \mathrm{ml}$ at admission) may predict a higher risk of endpoint events and a lower survival rate $(P<0.025)$.

\footnotetext{
* Correspondence: yhz2003333@163.com

†'Wei Pan, Donghui Yang and Peng Yu contributed equally to this work. ${ }^{1}$ Key laboratory of Geriatrics, Shengli Clinical Medical College of Fujian

Medical University, Fujian Institute of Clinical Geriatrics, No.134, Road Dongjie,

District Gulou, Fuzhou 350001, P.R. China

${ }^{4}$ Department of medicine, Fujian Provincial Hospital South Branch, Fuzhou 350028, China

Full list of author information is available at the end of the article
}

(c) The Author(s). 2020 Open Access This article is licensed under a Creative Commons Attribution 4.0 International License, which permits use, sharing, adaptation, distribution and reproduction in any medium or format, as long as you give appropriate credit to the original author(s) and the source, provide a link to the Creative Commons licence, and indicate if changes were made. The images or other third party material in this article are included in the article's Creative Commons licence, unless indicated otherwise in a credit line to the material. If material is not included in the article's Creative Commons licence and your intended use is not permitted by statutory regulation or exceeds the permitted use, you will need to obtain permission directly from the copyright holder. To view a copy of this licence, visit http://creativecommons.org/licenses/by/4.0/ The Creative Commons Public Domain Dedication waiver (http://creativecommons.org/publicdomain/zero/1.0/) applies to the data made available in this article, unless otherwise stated in a credit line to the data. 
(Continued from previous page)

Conclusions: The circulating biomarkers SST2, MMP-2 and NT-proBNP were all independent risk factors for patients with heart failure. The sST2 can be a useful biomarker with both diagnostic and prognostic value in patients with HFmrEF. The higher sST2 level in patients with heart failure was related to a higher incidence of combined endpoint outcome.

Keywords: HF, sST2, MMPs, NT-proBNP, Predictive value

\section{Background}

China is stepping into an aging society at present. The prevalence of heart failure (HF) become a serious problem due to the aging of population and the increased survival rate of patients with cardiovascular diseases. HF is a growing epidemic problem because of the significant morbidity and mortality rate [1]. At present, clinical diagnosis of HF is mainly based on medical history, physical signs and auxiliary examinations. Though in recent years, studies have been published on the aid of certain biomarkers in the diagnosis of HF, no ideal biomarker have been established to be widely used in clinical practice. In clinical practice, the plasma level of $\mathrm{N}$ terminal of the prohormone brain natriuretic peptide (NT-proBNP) is considered to be a good reference in the diagnosis and prognosis of HF with reduced ejection fraction (HFrEF). However, NT-proBNP is susceptible to many non-cardiac factor [2], which presented to have some limitations in the diagnosis of HF with mid-range ejection fraction (HFmrEF) and HF with preserved ejection fraction (HFpEF) [3]. Therefore, it is particularly important to explore better biomarkers with high sensitivity and specificity for HFmrEF and HFpEF.

Recently, soluble suppression of tumorigenicity 2 (sST2), matrix metalloproteinase (MMP)-2 and MMP-9 which are associated with cardiac remodeling and tissue fibrosis were reported to apply for the risk assessment in HF patients from western countries. Therefore, sST2 is considered to be the most valuable biomarker after NTproBNP in HF stratification recommended by guidelines $[4,5]$. However, there is still few comparative study on the diagnostic value of sST2, MMPs and NT-proBNP in Asian HF patients with different ejection fraction. So our study was to analyze the characteristic and clinical significance of these circulating blood biomarkers sST2, MMPs and NT-proBNP in HFpEF, HFmrEF and HFrEF patients. By comparing the diagnostic value of all these biomarkers, we may provide a more selective and effective detection for HF patients with different ejection fraction in clinical application.

\section{Methods}

\section{Study population}

A total of 163 consecutive patients with cardiac dysfunction who were hospitalized in Fujian Provincial Hospital from December 2016 to March 2018 were screened to be enrolled in the present study, including 85 males and 78 females, aged 42-86 years old. 163 patients were classified into four groups according to current ACC/AHA guidelines [6]: 50 cases in NYHA class I group, 24 cases in NYHA class II group, 53 cases in NYHA class III group, and 36 patients in NYHA class IV group. According to the standard of left ventricular ejection fraction measured by Cardiac Doppler ultrasound [3], 113 patients in NYHA class from II-IV grade were further divided into three subgroups: 60 cases with HFpEF group (LVEF $\geq 50 \%), 28$ cases with HFmrEF group (41 $\leq$ LVEF $\leq 49 \%$ ), 25 cases with HFrEF group (LVEF $\leq 40 \%$ ). General data such as age, gender, body mass index, blood pressure, blood lipids and renal function were collected. After admission, they were given conventional anti-heart failure medications. All subjects signed the informed consent. The patients who suffered from severe infection, pulmonary embolism, stroke, acute trauma, autoimmune diseases, hematopoietic diseases, malignant tumors, rheumatism, connective tissue diseases, pregnancy and other diseases affecting the secretion of SST2, MMP-2, MMP-9, and mental diseases or with the incomplete data affects the judge were excluded.

\section{Measurement of various indicators}

Height, weight, and blood pressure were measured in all subjects and body mass index (BMI) was calculated. $5 \mathrm{ml}$ of fast median cubital venous blood samples were collected the morning after admission. Sodium, blood sugar, blood lipids, and renal functions were measured by fiduciary institutions in our hospital.

\section{Echocardiographic data}

The routine color echocardiography was performed by the ultrasound specialist in the ultrasound room of Fujian Provincial Hospital. The patients were placed in the left lateral position, the probe was placed on the apex cordis, 2-dimensionally directed left ventricular (LV) Mmode dimensions were acquired from the parasternal long axis and carefully obtained perpendicular to the LV long axis and measured at the level of the mitral valve leaflet tips at end-diastole following the recommendations of the American Society of Echocardiography. LV end-systolic volume and LV ejection fraction (LVEF) 
were calculated using modified Simpson's method. Diastolic function was assessed by 2D and Doppler methods. From the apical four chamber view with color flow imaging, the indoor diameter and left ventricular end-diastolic volume, interval and thickness of the left and right ventricular wall and movement were observed. The structure of each valve was observed and the blood flow spectrum in the diastolic period, the acceleration and deceleration time of $E$ wave and a ratio of $E$ wave and $\mathrm{A}$ wave (E/A ratio), and the peak early diastolic flow velocity $(E)$, maximum speed of $E$ and peak late diastolic flow velocity $(\mathrm{A})$ were recorded from the mitral valve inflow velocity curve using pulsed wave Doppler at the tips of the mitral valve leaflet. The above measurements are the average of three cardiac cycles' measurements.

\section{Detection methods and procedures of main observation indicators}

The $5 \mathrm{ml}$ of fasting venous blood samples from subjects were collected in the Ethylene Diamine Tetraacetic Acid (EDTA)-K2 anti-coagulation tube on the next day after admission. The samples were centrifuged at $3000 \mathrm{r} / \mathrm{min}$ for $15 \mathrm{~min}$, and the plasma was extracted and transferred The $5 \mathrm{ml}$ of fasting venous blood samples from subjects were collected in the EDTA-K2 anti-coagulation tube on the next day after admission. The samples were centrifuged at $3000 \mathrm{r} / \mathrm{min}$ for $15 \mathrm{~min}$, and the plasma was extracted and saved in the $-70^{\circ} \mathrm{Crefrigerator}$. Samples were tested at the same time: SST2 kit was purchased from Wuhan Boshide Biological Company (No. EK1116), MMP-2 and MMP-9 kits were purchased from Hailian Biological Company (No. m19027652, m19026201). The plasma NT-proBNP concentration was determined by electrochemical luminescence immunoassay. The instrument was Elecsys 2010 (No. SLS-105) from Roche, Japan, and the experiment was completed by the Laboratory of Fujian Provincial Hospital. The indicators were tested strictly in accordance with the operating procedures of the kit instructions.

\section{Follow up}

During the following 1-3 years after discharge, patients were followed up by telephone to record adverse events, including all-cause death, re-admission for heart failure, and combined endpoint for heart failure. All-cause death is defined as death due to cardiovascular events (acute myocardial infarction, stroke, cardiogenic shock, etc.) or non-cardiovascular events (tumor, trauma, respiratory failure, etc.) Re-admission for heart failure was defined as a patient hospitalized for HF or spent more than $24 \mathrm{~h}$ in the emergency room. Some patients who were lost to follow-up were defined as the patient who was unable to find the ID number or the ID number was wrong, whose data were treated as the censored value in the final survival analysis.

\section{Statistical methods}

All data were processed by using SPSS 22.0 statistical software. Descriptive analyses are presented as mean \pm standard deviation for variables with normal distribution. The variables with non-normal distribution were expressed as the median (interquartile range). Study groups were compared using the Mann-Whitney $U$ test and the Kruskal-Wallis $\mathrm{H}$ test for data that did not present normal distribution, and the Nemenyi method was used for further comparison between two groups. Categorical variables were compared using chi-square tests. The receiver operating characteristic curve (ROC) was drawn to calculate the area under the curve (AUC) to evaluate the diagnostic value of the marker for heart failure. Correlation analysis between variables, continuous variables that presented normal distribution were analyzed by Pearson correlation, and variables that did not present normal distribution were analyzed by Spearman correlation. Spearman correlation analysis was used to describe the correlation between indicators. Logistic regression was used to analyze the risk factors for heart failure. The Kaplan-Meier survival curve was used to assess the incidence of adverse events in patients.

\section{Results}

\section{Basic data of HF patients}

113 patients were enrolled in this study. The average age of the participants was.

$69.92 \pm 13.94$ years old. Male patients accounted for 54.9\%, HFpEF, HFmrEF and HFrEF patients accounted for $53.1,24.8$ and $22.1 \%$, respectively. About $41.6 \%$ cases were $\mathrm{HF}$ accompanied with coronary artery disease, while $62.8 \%$ cases were HF combined with hypertension. Other HF patients combined with diabetes mellitus, atrial fibrillation accounted for $40.7,45.1 \%$, respectively. See Table 1.

\section{General information of HF patients with different ejection fractions}

Compared with indicators among HFpEF, HFmrEF and HFrEF groups, the results found that age, systolic blood pressure were statistically different among groups $(P<$ $0.05)$, but there was no significant difference among three groups in gender, BMI, diastolic blood pressure, triglycerides, total cholesterol, Low density lipoproteincholesterol (LDL-C), the rate of each HF phenotype taking HF treatment $(P>0.05)$ and other indicators, see Table 2. 
Table 1 Basic data of HF patients

\begin{tabular}{ll}
\hline Project & Basic data \\
\hline Age (years old) & $69.92 \pm 13.94$ \\
Male [Case (\%)] & $62(54.9 \%)$ \\
Type of HF [Case (\%)] & \\
HFpEF & $60(53.1 \%)$ \\
HFmrEF & $28(24.8 \%)$ \\
HFrEF & $25(22.1 \%)$ \\
Premedication history [Case (\%)] & \\
Cardiotonic & $36(31.9 \%)$ \\
Diuretic & $75(66.4 \%)$ \\
Aldosterone receptor antagonist & $31(27.4 \%)$ \\
$\quad$ ACEl/ ARB & $42(38.1 \%)$ \\
$\quad$ B receptor blocker & $32(28.3 \%)$ \\
Calcium channel antagonist & $40(35.4 \%)$ \\
Aspirin/Clopidogrel & $44(38.9 \%)$ \\
Statins & $46(40.7 \%)$ \\
Complication [Case (\%)] & \\
Coronary heart disease [Case (\%)] & \\
Hypertension [Case (\%)] & $47(41.6 \%)$ \\
Diabetes Mellitus [Case (\%)] & $71(62.8 \%)$ \\
Smoke History [Case (\%)] & $46(40.7 \%)$ \\
\hline
\end{tabular}

Note: ACEI Angiotensin Converting Enzyme Inhibitor, ARB Angiotensin Receptor Blocker

\section{Comparison of biomarker levels in HF patients with different ejection fractions}

The levels of plasma sST2 in HFpEF, HFmrEF, HFrEF patients were $1.31(0.30,2.80), 1.31(0.30,2.80)$ and 5.26 (2.82, 7.56) $\mathrm{ng} / \mathrm{ml}$, respectively. The plasma MMP-2 levels in HFpEF, HFmrEF, HFrEF patients were 3.81 $(2.50,5.75), 4.01(2.31,8.85)$ and $7.68(3.24,7.56) \mathrm{ng} / \mathrm{ml}$, respectively. The plasma MMP-9 levels of HFpEF, HFmrEF, HFrEF patients were 9.73 (3.69, 20.08), 11.39 $(6.58,26.10)$ and $22.43(7.95,32.46) \mathrm{ng} / \mathrm{ml}$, respectively. The levels of plasma NT-proBNP in HFpEF, HFmrEF, HFrEF patients were 2346.50 (838.77, 8164.00), 4536.00 $(921.90,9220.00)$ and $5934.00(2871.50,15,520.50) \mathrm{pg} /$ $\mathrm{ml}$, respectively, with the decrease of ejection fraction, the levels of these biomarkers showed an upward trend and it presented a statistical significance $(P<0.01)$.

Further multiple comparisons of plasma sST2 levels among the groups revealed that there is significant difference between every two groups $(P<0.01)$, as shown in Fig. 1. The plasma MMP-2 levels were significantly higher in the HFrEF group than in HFmrEF group and the HFpEF group $(P<0.01)$, but there was no statistical difference between' HFpEF and HFmrEF groups $(P>$ 0.05), as shown in Fig. 2. The plasma MMP-9 and NTproBNP levels were significantly higher in the HFrEF group than in the HFmrEF group $(P<0.01)$, but there is no statistical difference between HFpEF and HFmrEF group in MMP-9 and NT-proBNP levels $(P>0.05)$, there is no statistical difference between HFrEF and HFmrEF group in MMP-9 and NT-proBNP levels either $(P>0.05)$, as shown in Figs. 3 and 4.

\section{Diagnostic value of biomarkers in HFpEF patients}

In this study, the ROC curve was used to analyze the diagnostic value of plasma SST2, MMP-2, MMP-9 and NT-proBNP in HFpEF. The results suggested that NTproBNP had a higher AUC for the diagnosis of HFpEF than SST2, MMP-2 and MMP-9, and its sensitivity and specificity were also higher. The AUC of plasma sST2 for diagnosis of HFpEF was 0.717 (95\% confidence interval: $0.628-0.796, P<0.01)$, the optimal cut-off value was $0.332 \mathrm{ng} / \mathrm{ml}$, the sensitivity was $51.7 \%$, and the specificity was $95 \%$. The AUC of plasma MMP-2 for diagnosis of HFpEF was 0.705 (95\% confidence interval: 0.615-0.785, $P<0.01)$. The optimal cut-off value was $3.138 \mathrm{ng} / \mathrm{ml}$, the sensitivity was $55 \%$, and the specificity was $83.3 \%$. The AUC of plasma MMP-9 for diagnosis of HFpEF was 0.597 (95\% confidence interval: 0.504-0.686, $P<0.01$ ), the optimal cut-off value was $13.111 \mathrm{ng} / \mathrm{ml}$, the sensitivity was $41.7 \%$, and the specificity was $90 \%$. The AUC of plasma NT-proBNP for diagnosis of HFpEF was 0.881 (95\% confidence interval: $0.809-0.933, P<0.01$ ). The point value was $799.750 \mathrm{pg} / \mathrm{ml}$, the sensitivity was $78.3 \%$, and the specificity was $96.7 \%$, as shown in Fig. 5.

\section{Diagnostic value of bio-markers in HFmrEF patients}

In this study, the ROC curve was used to analyze the diagnostic value of plasma sST2, MMP-2, MMP-9 and NT-proBNP in HFmrEF. The results suggested that sST2 had a higher AUC for the diagnosis of HFmrEF than MMP-2, MMP-9 and NT-proBNP, and its sensitivity and specificity were also higher. The results showed that the AUC of plasma sST2 for diagnosis of HFmrEF was 0.799 (95\% confidence interval: $0.701-0.877, P<$ 0.01 ), the optimal cut-off value was $0.565 \mathrm{ng} / \mathrm{ml}$, the sensitivity was $92.9 \%$, and the specificity was $60.0 \%$. The AUC of plasma MMP-2 for diagnosis of HFmrEF was 0.678 (95\% confidence interval: $0.570-0.774, P<0.01$ ), the optimal cut-off value was $3.798 \mathrm{ng} / \mathrm{ml}$, the sensitivity was $60.7 \%$, and the specificity was $85 \%$. The AUC of plasma MMP-9 for diagnosis of HFmrEF was 0.676 (95\% confidence interval: $0.567-0.772, P<0.01)$, the optimal cut-off value was $15.049 \mathrm{ng} / \mathrm{ml}$, the sensitivity was $57.1 \%$, and the specificity was $96.7 \%$. The AUC of plasma NTproBNP for diagnosis of HFmrEF was 0.793 (95\% confidence interval: $0.694-0.872, P<0.01)$, the cut-off value is $898.1 \mathrm{pg} / \mathrm{ml}$, the sensitivity is $67.9 \%$, and the specificity is 98.3\%, as shown in Fig. 6. 
Table 2 Comparison of general data in HF patients with different ejection fractions

\begin{tabular}{|c|c|c|c|c|}
\hline Projects & $\begin{array}{l}\text { HFpEF group } \\
(\geq 50 \%, n=60)\end{array}$ & $\begin{array}{l}\text { HFmrEF group } \\
(41-49 \%, n=28)\end{array}$ & $\begin{array}{l}\text { HFrEF group } \\
(\leq 40 \%, n=25)\end{array}$ & $P$ value \\
\hline Age (year) & $73.35 \pm 11.31$ & $68.82 \pm 14.28$ & $62.92 \pm 16.79^{a}$ & 0.021 \\
\hline Male (case \%) & $26(43.3 \%)$ & $18(64.3 \%)$ & $18(72.0 \%)$ & 0.796 \\
\hline BMI $\left(\mathrm{kg} / \mathrm{m}^{2}\right)$ & $23.95 \pm 4.20$ & $22.98 \pm 3.03$ & $23.03 \pm 3.43$ & 0.499 \\
\hline $\begin{array}{l}\text { Systolic blood pressure } \\
(\mathrm{mm} \mathrm{Hg})\end{array}$ & $139.28 \pm 27.64$ & $139.44 \pm 22.51$ & $121.04 \pm 17.25^{\mathrm{ab}}$ & 0.011 \\
\hline Diastolic blood pressure (mmHg) & $76.07 \pm 13.92$ & $76.86 \pm 14.76$ & $78.20 \pm 13.73$ & 0.819 \\
\hline Serum sodium (mmol/L) & $140.60 \pm 3.95$ & $140.36 \pm 3.75$ & $141.32 \pm 3.02$ & 0.607 \\
\hline Fasting glucose (mmol/L) & $6.30 \pm 1.11$ & $6.54 \pm 1.19$ & $6.21 \pm 0.97$ & 0.504 \\
\hline LVEF (\%) & $57.19 \pm 5.43$ & $44.45 \pm 2.99$ & $33.23 \pm 3.98$ & $<0.01$ \\
\hline $\begin{array}{l}\text { Triglyceride } \\
(\mathrm{mmol} / \mathrm{L})\end{array}$ & $1.39 \pm 0.85$ & $1.40 \pm 0.74$ & $1.38 \pm 0.66$ & 0.832 \\
\hline Total cholesterol (mmol/L) & $4.04 \pm 1.14$ & $4.29 \pm 1.24$ & $4.25 \pm 1.08$ & 0.721 \\
\hline LDL-C (mmol/L) & $2.54 \pm 0.99$ & $2.76 \pm 1.08$ & $2.85 \pm 1.08$ & 0.432 \\
\hline $\begin{array}{l}\text { Serum Creatinine } \\
(\mu \mathrm{mol} / \mathrm{L})\end{array}$ & $132.08 \pm 20.63$ & $108.71 \pm 49.31$ & $104.12 \pm 44.78$ & 0.813 \\
\hline $\begin{array}{l}\text { eGFR } \\
\left(\mathrm{ml} / \mathrm{min} / 1.73 \mathrm{~m}^{2}\right)\end{array}$ & $65.52 \pm 3.94$ & $55.99 \pm 5.37$ & $53.20 \pm 8.48$ & 0.237 \\
\hline $\begin{array}{l}\text { ACEI/ARB } \\
\text { [case (\%)] }\end{array}$ & $22(36.7 \%)$ & $13(46.4 \%)$ & $7(28 \%)$ & 0.383 \\
\hline $\begin{array}{l}\beta \text {-blockers } \\
\text { [case (\%)] }\end{array}$ & 18 (30.0\%) & 7 (25.0\%) & 7 (28.0\%) & 0.889 \\
\hline MRA [case (\%)] & $12(20.0 \%)$ & $8(28.6 \%)$ & $11(44.0 \%)$ & 0.079 \\
\hline
\end{tabular}

Note: BMI Body Mass Index, LDL-C Low Density Lipoprotein Cholesterol, LVEF Left Ventricular Ejection Fraction, eGFR Estimated Glomerular Filtration Rate, 1 $\mathrm{mmHg}=0.133 \mathrm{kPa}$, a: Compared with HFpEF group, b: Compared with HFmrEF: ${ }^{*} P<0.05$

\section{Diagnostic value of bio-markers in HFrEF patients}

In this study, the ROC curve was used to analyze the diagnostic value of SST2, MMP-2, MMP-9 and NTproBNP in HFrEF. The results suggested that NTproBNP had a higher AUC for the diagnosis of HFrEF than sST2, MMP-2 and MMP-9, and its sensitivity and specificity were also higher. The results showed that the

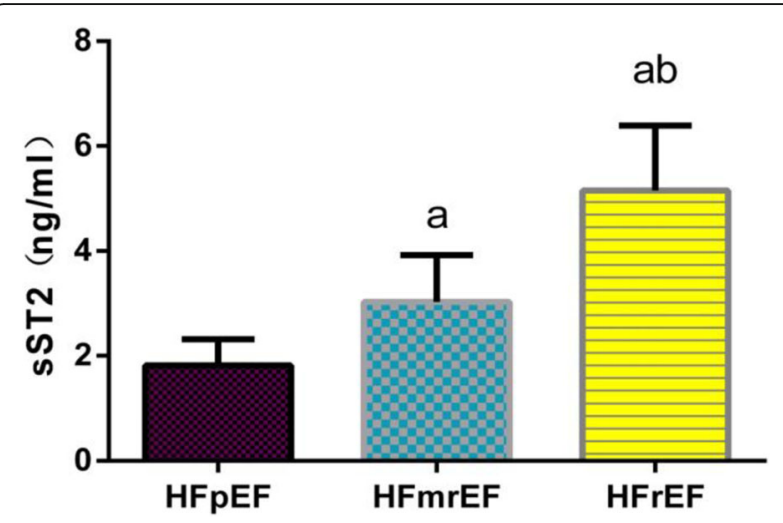

Fig. 1 Comparison of plasma sST2 levels among groups. Note: a: Compared with HFpEF group $P<0.01$, b: Compared with HFmrEF group $P<0.01$
AUC of plasma NT-proBNP for diagnosis of HFrEF was 0.945 (95\% confidence interval: 0.874-0.983, $P<0.01$ ), the cut-off value was $1106.700 \mathrm{pg} / \mathrm{ml}$, the sensitivity was $88.0 \%$, and the specificity was $98.3 \%$. The AUC of plasma sST2 for diagnosis of HFrEF was 0.820 (95\% confidence interval: $0.722-0.895, P<0.01)$, the optimal cutoff value was $2.539 \mathrm{ng} / \mathrm{ml}$, the sensitivity was $84.0 \%$, and

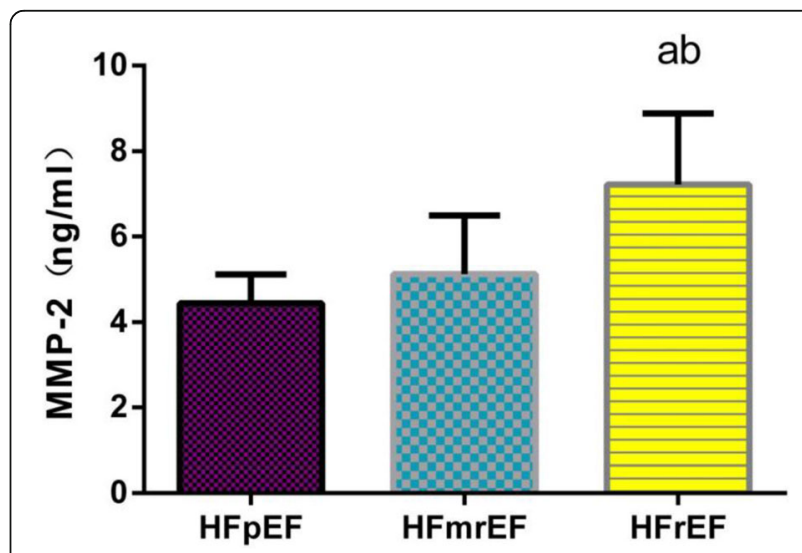

Fig. 2 Comparison of plasma MMP-2 levels among groups. Note: a: Compared with HFpEF group $P<0.01 \mathrm{~b}$ : Compared with HFmrEF group $P<0.01$ 


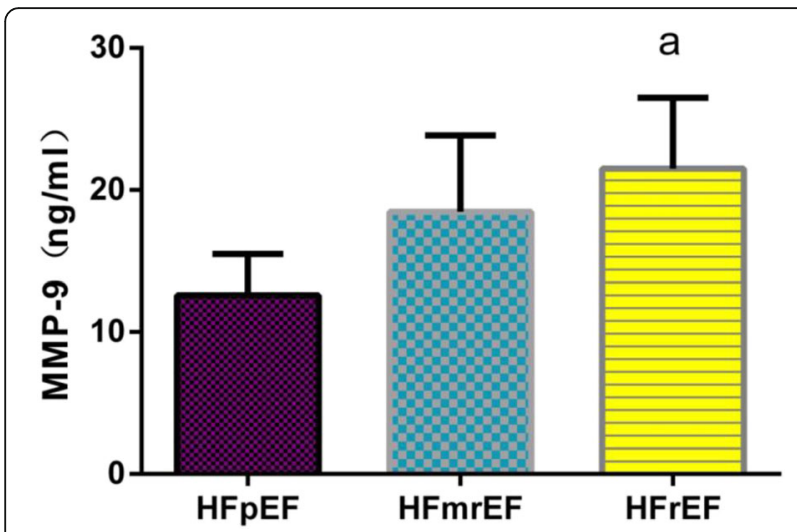

Fig. 3 Comparison of plasma MMP-9 levels among groups. Note: a: Compared with HFpEF group $P<0.01$

the specificity was $70.0 \%$. The AUC of plasma MMP-2 for diagnosis of HFrEF was 0.814 (95\% confidence interval: $0.715-0.890, P<0.01)$, the optimal cut-off value was $3.846 \mathrm{ng} / \mathrm{ml}$, the sensitivity was $72.0 \%$, and the specificity was $85.0 \%$. The AUC of plasma MMP-9 for diagnosis of HFrEF was 0.774 (95\% confidence interval: 0.670-0.858, $P<0.01$ ), the optimal cut-off value was $16.748 \mathrm{ng} / \mathrm{ml}$, the sensitivity was $68.0 \%$, and the specificity was $98.3 \%$, as shown in Fig. 7.

The relationship of plasma SST2 levels and related factors in HF patients with different ejection fractions

Spearman correlation analysis results suggest that the plasma SST2 levels are positively correlated with plasma MMP-2 ( $\mathrm{r}$ value were $+0.330,+0.505,+0.562$ respectively, $P<0.05$ ), MMP-9 ( $\mathrm{r}$ value were $+0.558,+0.376$, + $0.811, P<0.05$ ) and NT-proBNP ( $\mathrm{r}$ value were $+0.493,+$ $0.448,+0.688, P<0.05)$, regardless of the value of LVEF, as shown in Table 3.

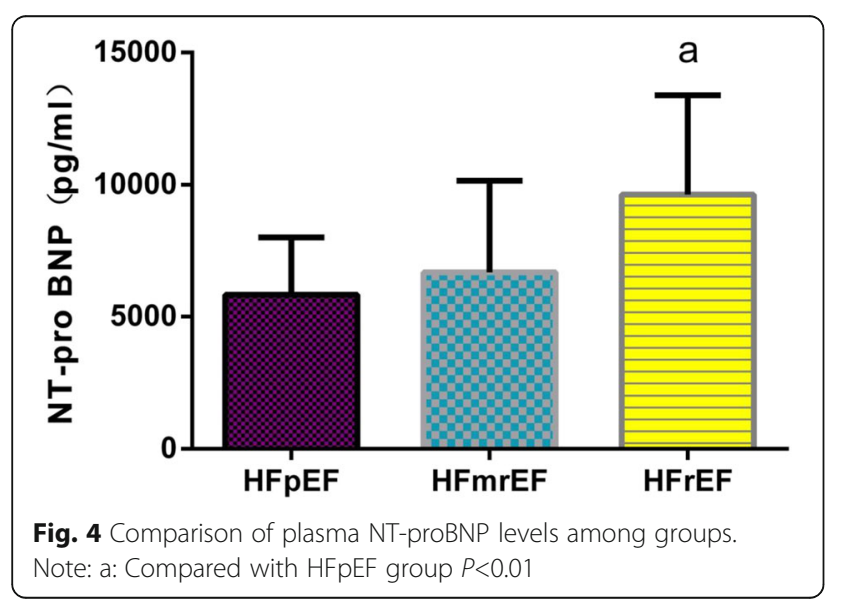

The analysis of affecting factors with HF

HF was taken as a dependent variable, while age, gender, LVEF, blood lipids, blood pressure, BMI, creatinine, sST2, MMP-2, MMP-9, and NT-proBNP were gradually entered into the model as an independent variable. The significance level was defined as 0.05 after the model was selected. Logistic regression analysis was performed after excluding the confounding factors and interaction effect. The results showed that the independent variables such as age, sST2, MMP-2 and NT-proBNP were independent risk factors for heart failure, as shown in Table 4.

\section{Total end-point events of HF during follow-up}

There were 113 subjects were enrolled in this study, six of them were lost to follow up, the loss ratio was 5.3\%. 32 patients were dead during a median follow-up of 778 days (CI: 540 days-1080 days). In our study, the all-cause mortality rate was $29.9 \%$, and rate of re-admission to HF was $38.3 \%$, incidence of combined endpoints was $53.2 \%$. In this study, re-admission rate of patients with $\mathrm{HFpEF}$, HFmrEF, and HFrEF during a median follow-up of 778 days were $23.3,39.2$ and $56.0 \%$ respectively, and the incidence rate of combined end points were 23.3, 56.2, and $75.1 \%$, respectively. All these data were statistically significant $(P<0.05$, as shown in Table 5).

\section{Effect of plasma sST2 baseline on endpoint events}

We compared the baseline of plasma SST2 to find its relationship with the risk of adverse events: re-admission or all-cause death to HF during a median follow-up of 778 days after discharge. The patients were divided into low-level group (sST2 $<0.332 \mathrm{ng} / \mathrm{ml}$ ) and high-level group (sST2 $\geq 0.332 \mathrm{ng} / \mathrm{ml}$ ) according to the value of sST2 which the optimal cut off value of plasma sST2 at admission was obtained through the ROC curve analysis of Fig. 8. Survival analysis indicated that the patients in the high-level sST2 group suffered from a higher risk of end-point events and a lower survival rate (log-rank chisquare value $=5.036, P=0.025$ ), as shown in Table 6 and Fig. 8.

\section{Discussion}

HF is the end stage of several pathological cardiac conditions and is currently the most common cause of death in cardiovascular diseases. Nowadays, the guidelines of HF in various countries mainly recommend brain natriuretic peptide (BNP) and NT-proBNP [2] to be a useful biomarker in the judgment of diagnosis, severity degree assessment, and prognostic evaluation of endpoint events for HF. Plasma NT-proBNP level is more sensitive than BNP when cardiac pressure or volume load increases, the half-life of NT-proBNP is also longer and more stable [7]. However, NT-proBNP can't correctly 


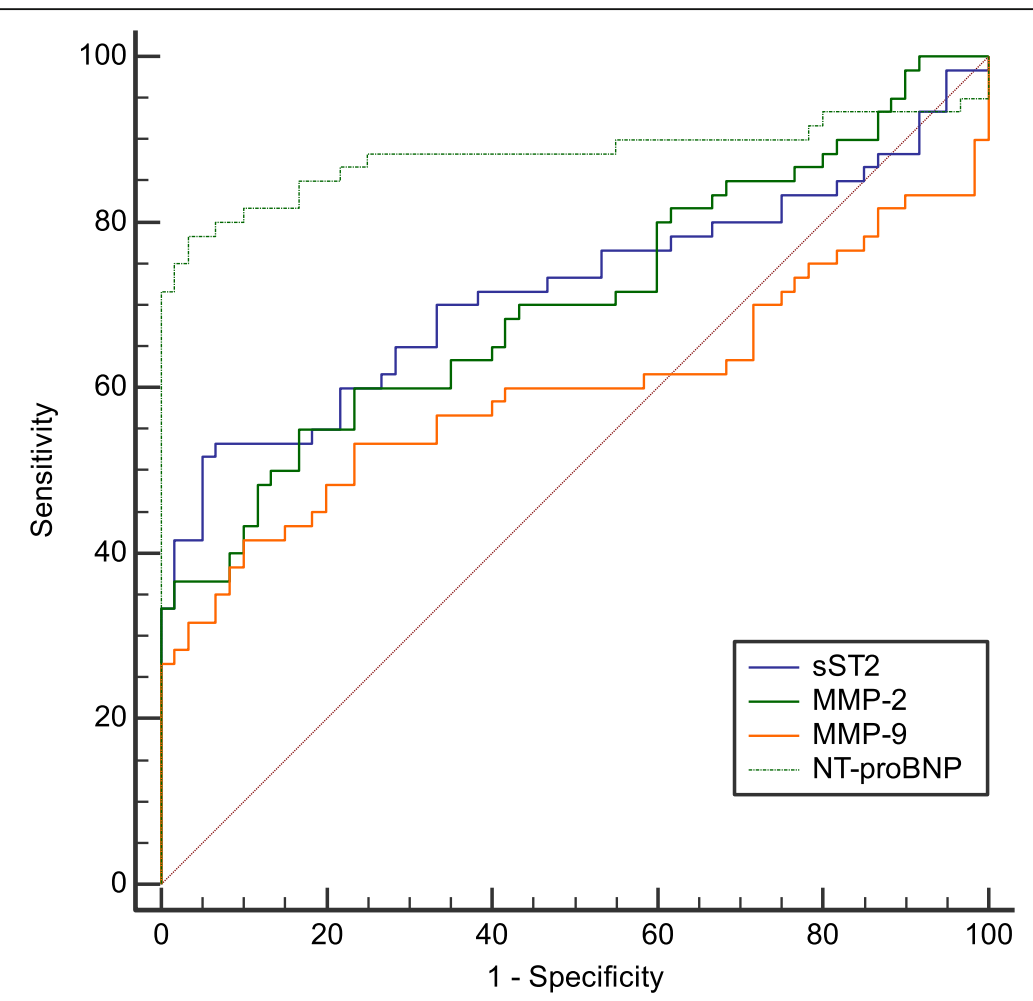

Fig. 5 ROC curve of biomarkers plasma sST2, MMP-2, MMP-9 and NT-proBNP for diagnosis of HFpEF

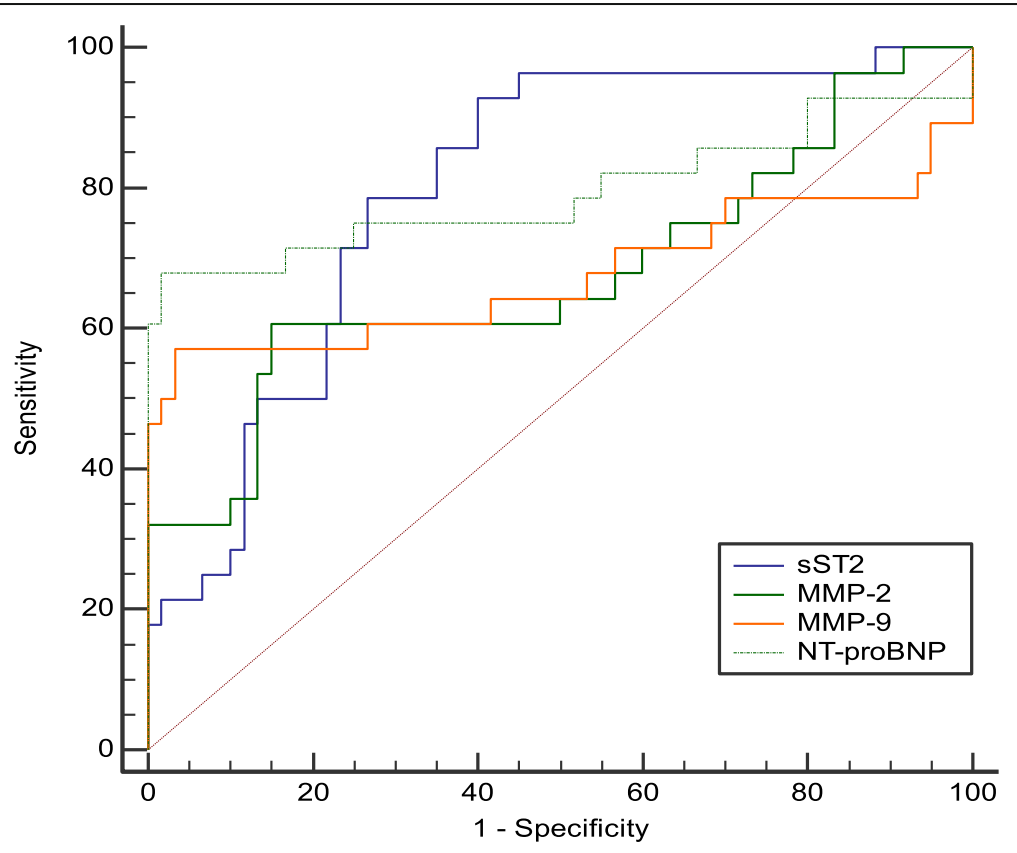

Fig. 6 ROC curve of biomarkers plasma SST2, MMP-2, MMP-9 and NT-proBNP for diagnosis of HFmrEF 


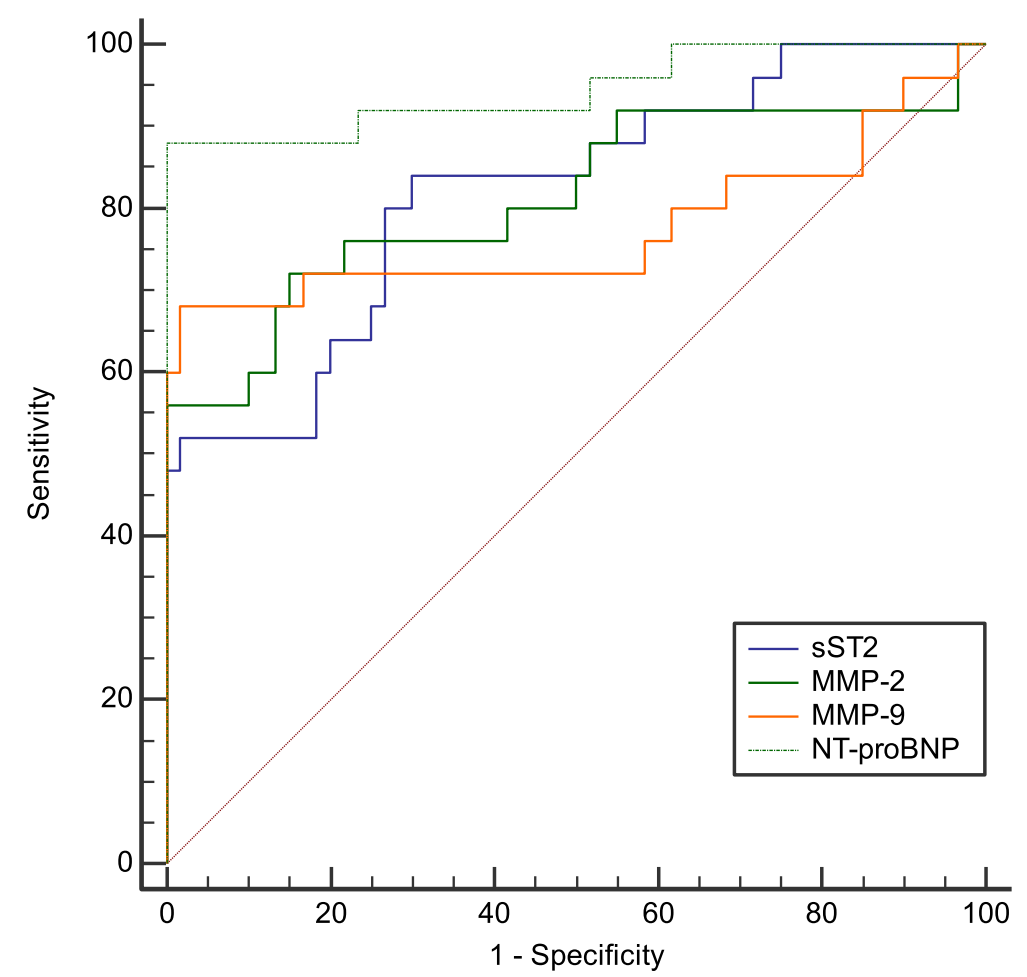

Fig. 7 ROC curve of biomarkers plasma sST2, MMP-2, MMP-9 and NT-proBNP for diagnosis of HFrEF

Table 3 Spearman correlation analysis of plasma sST2 levels and related factors in patients with HF

\begin{tabular}{|c|c|c|c|c|c|c|}
\hline \multirow{2}{*}{$\begin{array}{l}\text { Projects } \\
-\end{array}$} & \multicolumn{2}{|c|}{ HFpEF group $(n=60)$} & \multicolumn{2}{|c|}{ HFmrEF group $(n=28)$} & \multicolumn{2}{|c|}{ HFrEF group $(n=25)$} \\
\hline & $r$ & $P$ & $r$ & $P$ & $r$ & $P$ \\
\hline Age (Years old) & +0.058 & 0.685 & -0.087 & 0.660 & +0.106 & 0.614 \\
\hline Male [Case (\%)] & +0.185 & 0.276 & +0.240 & 0.219 & -0.046 & 0.670 \\
\hline BMI $\left(\mathrm{kg} / \mathrm{m}^{2}\right)$ & +0.028 & 0.833 & -0.087 & 0.660 & -0.055 & 0.792 \\
\hline $\begin{array}{l}\text { Diabetes Mellitus } \\
\text { [Case (\%)] }\end{array}$ & +0.191 & 0.075 & -0.118 & 0.462 & +0.059 & 0.727 \\
\hline $\begin{array}{l}\text { Atrial fibrillation } \\
\text { [Case (\%)] }\end{array}$ & +0.087 & 0.415 & +0.100 & 0.553 & +0.277 & 0.103 \\
\hline $\begin{array}{l}\text { Coronary heart disease } \\
\text { [Case }(\%)]\end{array}$ & +0.102 & 0.341 & -0.136 & 0.394 & +0.062 & 0.717 \\
\hline $\begin{array}{l}\text { Hypertension } \\
\text { [Case (\%)] }\end{array}$ & +0.120 & 0.263 & -0.132 & 0.441 & +0.289 & 0.089 \\
\hline Systolic blood pressure (mmHg) & -0.005 & 0.969 & +0.048 & 0.808 & +0.037 & 0.861 \\
\hline Diastolic blood pressure (mmHg) & -0.014 & 0.913 & +0.355 & 0.063 & +0.105 & 0.618 \\
\hline Triglyceride (mmol/L) & +0.05 & 0.705 & -0.101 & 0.609 & -0.072 & 0.733 \\
\hline Total cholesterol (mmol/L) & -0.132 & 0.314 & +0.252 & 0.196 & -0.251 & 0.226 \\
\hline LDL-C (mmol/L) & -0.090 & 0.496 & +0.360 & 0.060 & -0.231 & 0.132 \\
\hline $\begin{array}{l}\text { eGFR } \\
{\left[\mathrm{ml} /\left(\min \cdot 1.73 \mathrm{~m}^{2}\right)\right]}\end{array}$ & -0.117 & 0.726 & +0.008 & 0.969 & -0.195 & 0.351 \\
\hline MMP-2 (ng/ml) & +0.330 & 0.010 & +0.505 & $<0.05$ & +0.562 & 0.003 \\
\hline MMP-9 (ng/ml) & +0.558 & $<0.05$ & +0.376 & 0.001 & +0.811 & $<0.05$ \\
\hline NT-proBNP (pg/ml) & +0.493 & $<0.05$ & +0.448 & $<0.05$ & +0.688 & $<0.05$ \\
\hline
\end{tabular}


Table 4 Logistic regression analysis of factors affecting HF

\begin{tabular}{lllll}
\hline Projects & OR & Wald & $95 \% \mathrm{Cl}$ & $P$ value \\
\hline Age & 0.140 & 10.384 & $1.056-1.252$ & 0.001 \\
sST2 $(\mathrm{ng} / \mathrm{ml})$ & 1.960 & 3.797 & $1.409-35.758$ & 0.004 \\
MMP-2 $(\mathrm{ng} / \mathrm{ml})$ & 0.805 & 5.468 & $1.139-4.390$ & 0.019 \\
NT-proBNP $(\mathrm{pg} / \mathrm{ml})$ & 0.002 & 4.412 & $1.001-1.004$ & 0.036 \\
\hline
\end{tabular}

Note: $\mathrm{OR}$ Odds ratio, $\mathrm{Cl}$ confidence interval

reflect degree of left ventricular filling because the relatively longer half-life can be affected by the retention of fluid in the body [3]. Besides, NT-proBNP level was affected by many confounding factors, such as age, LV hypertrophy, renal insufficiency and dyspnea which frequently lead to false negative results and the wrong clinical judgment. At present, there is no ideal biomarker can be used for the early diagnosis, the severity and prognosis of HF and not affected by other non-cardiac factors.

It's reported that Trans mural stress stimulation could cause an increase in ventricular wall tension, thus, cardiomyocytes were mechanically stretched and stimulated to secrete NT-proBNP, as well as the sST2, MMP-2 and MMP-9. In the MOCA study, various biomarkers such as sST2, BNP, and NT-proBNP were evaluated, and they were all identified as independent risk factors [8]. Our study found that regardless of the level of LVEF, the sST2 levels in patients at admission were positively correlated with MMP-2, MMP-9 and NT-proBNP. sST2, MMP-2, and NT-proBNP were all independent risk factors of heart failure which can be used for risk stratification. This may be related to age-related vascular stiffness and intensified left ventricular wall tension. Recently, biomarkers related to myocardial remodeling, such as sST2 and MMPs, have been recommended by the international HF guidelines in the diagnosis and prognosis of HF. However, there is neither Asian report about their application, nor reports of large data in patients with HFmrEF. Therefore, in our study, we focused on the NT-proBNP, sST2 and MMPs, and compared the diagnostic and prognostic value of these biomarkers in HF patients with different ejection fraction for precision diagnosis of $\mathrm{HF}$.

Table 5 Main endpoint of HFpEF, HFmrEF and HFrEF patients during a median follow-up of 778 days

\begin{tabular}{lllll}
\hline Endpoint (\%) & HFpEF & HFmrEF & HFrEF & $P$ \\
\hline All-cause mortality & 16.6 & 35.7 & 48.0 & 0.124 \\
Heart failure readmission & 23.3 & 39.2 & 56.0 & 0.013 \\
Combined endpoint & 31.3 & 56.2 & 75.1 & 0.008 \\
\hline Note: Combined end point: all-cause mortality and/or re-admission of HF
\end{tabular}

SANDERS-VAN et al. [9] scholars found that patients with HFrEF had higher levels of plasma NT-proBNP than patients with $\mathrm{HFpEF}$, and the correlation in HFrEF was also higher than in HFpEF. This may owe to lower left ventricular pressure in HFpEF patients. Our study observed that the plasma NT-proBNP concentration gradually increased with the decrease of LVEF, besides, plasma NT-proBNP levels in HFrEF patients were significantly higher than in HFpEF patients, which is consistent with previous studies [10]. In the diagnostic value assessment of the four biomarkers for three types of heart failure, the AUC for the diagnosis of HFpEF and HFrEF by plasma NT-proBNP is higher than that of plasma sST2, MMP-2, and MMP-9, suggesting that the diagnostic predictive value of plasma NT-proBNP for HFpEF and HFrEF is better than that of other markers.

Cardiac collagen remodeling is important in the progression of heart failure. Serum markers of cardiac extracellular matrix (ECM) turnover proteins such as MMPs are positively correlated with interstitial fibrosis, diastolic dysfunction and left ventricular hypertrophy [10, 11]. MMP-2 and MMP-9 are important components of ECM. In a study of 62 patients with chronic HF, MMP-2 levels were reduced in patients with significant LVEF recovery after treatment with $\beta$ blockers, while MMP-2 levels were elevated in patients with less improved LVEF [12]. Our results showed that the plasma levels of MMP2 and MMP-9 were significantly higher in the HFrEF group than in HFmrEF and HFpEF group $(P<0.01)$, but there was no statistical difference between HFpEF and HFmrEF groups $(P>0.05)$. Further Spearman correlation analysis suggested that the MMP-2 but not MMP-9 could be used as an independent risk factors for heart failure diagnosis, which was consistent with the results of George J's study [13]。 .

ST2 is a member of the interleukin 1 receptor family and exists in two forms, a trans-membrane receptor (ST2L) as well as a soluble decoy receptor (sST2) [14]. The ligand of ST2 is Interleukin-33 (IL-33), which is involved in reducing fibrosis and hypertrophy in mechanically strained tissues. Overexpression of sST2 was significantly related to poor myocardial remodeling, cardiac insufficiency, and hemodynamic abnormalities [15]. Tseng et al. found that the patients in the endstage of HF had a higher sST2 level compared with the patients in the NYHA stage II and III of HF, but the level could decrease within 3 months after implantation of left ventricular assist device [16]. Shah et al. [17] found that sST2 levels were significantly associated with abnormal changes in function and structure such as ventricular enlargement, LV diastolic dysfunction etc. In our study, Spearman correlation analysis results indicated that sST2 could be an independent risk factor for heart failure diagnosis. Besides, we found there is a 


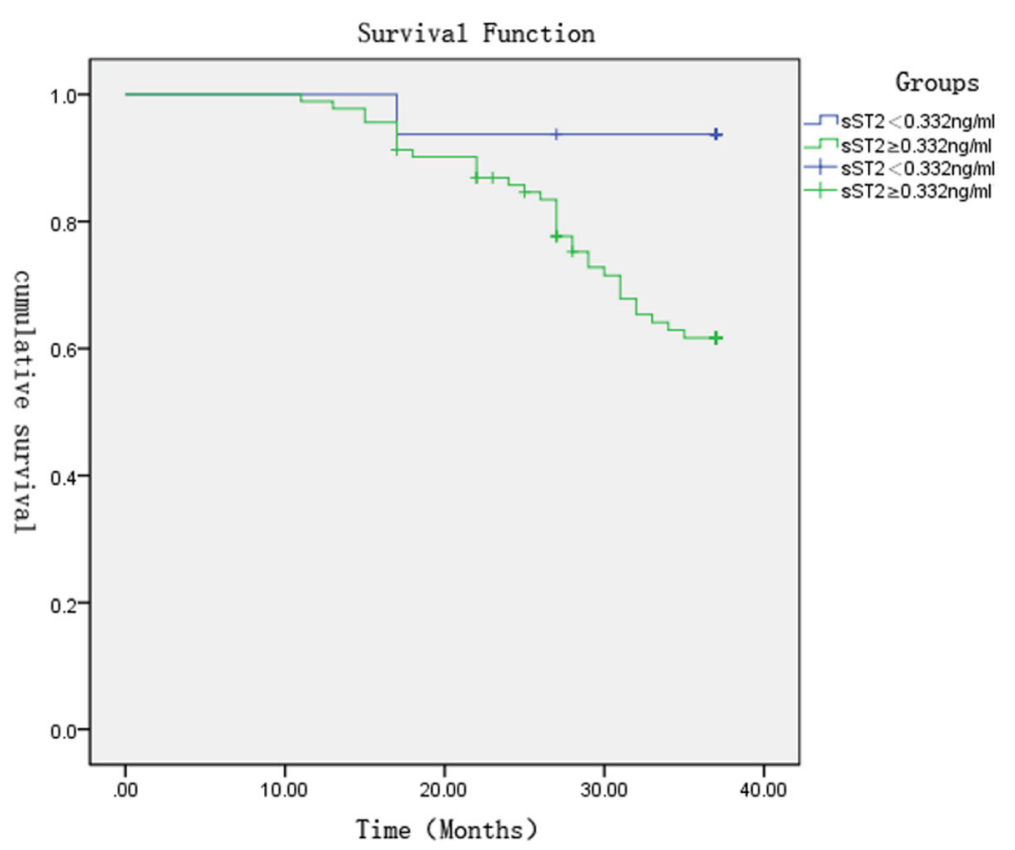

Fig. 8 Survival analysis of SST2 baseline levels at admission

statistical difference when compared the sST2 levels in HFmrEF and HFpEF patients, the area under the ROC curve for the diagnosis of HFmrEF by plasma sST2 levels is significantly higher than that of plasma MMPs and NT-proBNP, and its sensitivity and specificity are both higher, suggesting that the independent diagnostic value of plasma sST2 levels for HFmrEF is better than that of plasma NT-proBNP and MMPs. It can be read that SST2 is a more accurate marker for the diagnosis of HFmrEF in the "grey zone" of heart failure patients, which is also consistent with previous studies [18]. Further survival analysis of our study indicated that the HF patients in the base high-level sST2 suffered from a higher risk of end-point events and a lower survival rate. This result proved the same as Vark's study. We can read that in terms of prognostic value of HF, sST2 is considered to be a better predictor than NT-proBNP and MMPs [19].

Since this study did not analyze indicators associated with diastolic function in echocardiography, it is necessary to further study related factors in the subsequent studies.

Table 6 Comparison of survival status under plasmas ST2 baseline levels

\begin{tabular}{llllll}
\hline Indicator & & Survival rate & Mortality rate & $c^{2}$ & $P$ \\
\hline SST2 $(\mathrm{ng} / \mathrm{ml})$ & $<0.332$ & $93.7 \%$ & $6.3 \%$ & 5.036 & 0.025 \\
& $\geq 0.332$ & $64.1 \%$ & $35.9 \%$ & & \\
\hline
\end{tabular}

\section{Conclusion}

The markers sST2, MMP-2 and NT-proBNP are all independent risk factors for patients with heart failure. The plasma sST2 levels can be a useful marker with both diagnostic and prognostic values in patients with HFmrEF. The higher the plasma sST2 level in patients with heart failure was related to a higher incidence of combined endpoint outcome.

\section{Abbreviations}

NT-proBNP: N-terminal of the prohormone brain natriuretic peptide; sST2: Soluble Supperssion of tumorigenicity 2; MMPs: Matrix metalloproteinases; HF: Heart failure; HFpEF: HF with preserved ejection fraction; HFmrEF: HF with mid-range ejection fraction; HFrEF: HF with reduced ejection fraction; ELISA: Enzyme linked immunosorbent assay; ROC: Receiver operating characteristic; AUC: The area under the curve; OR: Odds ratio; BMI: Body mass index; LV: Left ventricular; LVEF: LV ejection fraction; EDTA: Ethylene Diamine Tetraacetic acid; ACEl: Angiotensin converting enzyme inhibitors; ARB: Angiotensin receptor blocker; LDL-C: Low density lipoprotein-cholesterol; eGFR: Estimated glomerular filtration rate; ECM: Extracellular matrix; IL-33: Interleukin-33; Cl: Confidence interval; BNP: Brain natriuretic peptide

\section{Acknowledgements}

The authors would like to thank other colleagues whom were not listed in the authorship of Key laboratory of Geriatrics, Shengli Clinical Medical College of Fujian Medical University and Sun Yat-sen University for their helpful comments on the manuscript.

\section{Authors' contributions}

HY and WP conceived and designed the experiments; DY recruited subjects and collected clinical data. PY conducted the laboratory testing. WP, DY and PY helped to analyze the data. HY and WP wrote the manuscript. All authors read and approved the final manuscript. 


\section{Funding}

This work was supported by grants from the National Natural Science Foundation of China (No. 81670258, 81873515), the youth scientific research project of Fujian provincial health commission (No. 20170132) and Science and Technology Planning Project of Shenzhen Municipality (No.JCYJ20180306173433984). The funders are HY and WP, they played important role in the design of the study and collection, analysis, and interpretation of data and in writing the manuscript.

\section{Availability of data and materials}

The datasets used and/or analyzed during the current study are de-identified and available from the corresponding author on reasonable request.

\section{Ethics approval and consent to participate}

The study was approved by the Ethics Committee of Fujian Provincial Hospital, (NO: K2019-05-032). Written informed consent was obtained from each patient.

\section{Consent for publication}

Not applicable.

\section{Competing interests}

The authors declare that they have no competing interests.

\section{Author details}

${ }^{1}$ Key laboratory of Geriatrics, Shengli Clinical Medical College of Fujian Medical University, Fujian Institute of Clinical Geriatrics, No.134, Road Dongjie, District Gulou, Fuzhou 350001, P.R. China. ${ }^{2}$ Department of cardiology, The Eighth Affiliated Hospital, Sun Yat-Sen University, Shenzhen 518000, China. ${ }^{3}$ Zhengzhou People's Hospital, Zhengzhou 450000, China. ${ }^{4}$ Department of medicine, Fujian Provincial Hospital South Branch, Fuzhou 350028, China.

Received: 15 September 2019 Accepted: 21 April 2020

Published online: 30 April 2020

\section{References}

1. Han YL. Follow the current guideline: optimizing the diagnosis, treatment and management of heart failure. Zhonghua Xin Xue Guan Bing Za Zhi. 2018;46(10):753-5.

2. Karayannis G, Triposkiadis F, Skoularigis J, et al. The emerging role of Galectin-3 and ST2 in heart failure: practical considerations and pitfalls using novel biomarkers. Curr Heart Fail Rep. 2013;10(4):441-9.

3. Ponikowski P, Voors AA, et al. 2016 ESC guidelines for the diagnosis and treatment of acute and chronic heart failure: the task force for the diagnosis and treatment of acute and chronic heart failure of the European Society of Cardiology. Eur J Heart Fail. 2016;18(8):891-975.

4. Chinese Society of Cardiology, Chinese Journal of Cardiology. Chinese guidelines for the diagnosis and treatment of heart failure. Chin J Cardiol. 2014;42(2):98-122.

5. Yancy CW, Jessup M, Bozkurt B, et al. 2017 ACC/AHA/HFSA focused update of the 2013 ACCF/AHA guideline for the Management of Heart Failure: a report of the American College of Cardiology/American Heart Association task force on clinical practice guidelines and the Heart Failure Society of America. Circulation. 2017;136(6):e137-61.

6. Hunt SA, Abraham WT, Chin MH, et al. ACC/AHA 2005 guideline update for the diagnosis and management of chronic heart failure in the adult: a report of the American College of Cardiology/American Heart Association Task Force on Practice Guidelines (Writing Committee to Update the 2001 Guidelines for the Evaluation and Management of Heart Failure):developed in collaboration with the American College of Chest Physicians and the International Society for Heart and Lung Transplantation: endorsed by the Heart Rhythm Society. Circulation. 2005;112(12):e154-235.

7. Tominaga S. A putative protein of a growth specific $c$ DNA from BALB/c3T3 cells is highly similar to the extracellular portion of mouse interleukin 1 receptor. FEBS Lett. 1989;258(2):301-4.

8. Lassus J, Gayat E, Mueller C, et al. Incremental value of biomarkers to clinical variables for mortality prediction in acutely decompensated heart failure: the multinational observational cohort on acute heart failure (MOCA) study. Int J Cardiol. 2013;168(3):2186-94.
9. Sanders-van Wijk S, van Empel V, Davarzani N, et al. Circulating biomarkers of distinct pathophysiological pathways in heart failure with preserved vs reduced ventricular ejection fraction. Eur J Heart Fail. 2015;17(10):1006-14.

10. Manhenke $\mathrm{C}$, Ueland $\mathrm{T}$, Jugdutt $\mathrm{Bl}$, et al. The relationship between markers of extracellular cardiac matrix turnover: infarct healing and left ventricular remodelling following primary $\mathrm{PCl}$ in patients with first-time STEMI. Eur Heart J. 2014;35(6):395-402.

11. Zile MR, Desantis SM, Baicu CF, et al. Plasma biomarkers that reflect determinants of matrix composition identify the presence of left ventricular hypertrophy and diastolic heart failure. Circ Heart Fail. 2011;4(3):246-56.

12. Fonarow GC, Abraham WT, Albert NM, et al. Influence of beta-blocker continuation or withdrawal on outcomes in patients hospitalized with heart failure: findings from the OPTIMIZE-HF program. J Am Coll Cardiol. 2008; 52(3):190-9

13. Fukui M, Goda A, Komamura K, et al. Changes in collagen metabolism account for ventricular functional recovery following beta-blocker therapy in patients with chronic heart failure. Heart Vessel. 2016;31(2):173-82.

14. George J, Patal S, Wexler D, et al. Circulating matrix metalloproteinase-2 but not matrix metalloproteinase-3, matrix metalloproteinase-9, or tissue inhibitor of metalloproteinase-1 predicts outcome in patients with congestive heart failure. Am Heart J. 2005;150(3):484-7.

15. Iwahana $\mathrm{H}$, Yanagisawa $\mathrm{K}$, Ito-Kosaka $\mathrm{A}$, et al. Different promoter usage and multiple transcription initiation sites of the interleukin-1 receptor- related human ST2 gene in UT-7 and TM12 cells. Eur J Biochem. 1999;264(2):397406.

16. Schmitz J, Owyang A, Oldham E, et al. IL-33, an interleukin-1-like cytokine that signals via the IL-1 receptor-related protein ST2 and induces T helper type 2-associated cytokines. Immunity. 2005;23(5):479-90.

17. Tseng CCS, Huibers MMH, Gaykema LH, et al. Soluble ST2 in end-stage heart failure, before and after support with a left ventricular assist device. Eur J Clin Investig 2018;48(3):e12886.

18. Michalska-Kasiczak M, Bielecka-Dabrowa A, von Haehling S, et al. Biomarkers, myocardial fibrosis and co-morbidities in heart failure with preserved ejection fraction: an overview. Arch Med Sci. 2018;14(4):890-909.

19. Van Vark LC, Lesman-Leegte I, Baart SJ, et al. Prognostic value of serial ST2 measurements in patients with acute heart failure. J Am Coll Cardiol. 2017; 70(19):2378-88.

\section{Publisher's Note}

Springer Nature remains neutral with regard to jurisdictional claims in published maps and institutional affiliations.

Ready to submit your research? Choose BMC and benefit from:

- fast, convenient online submission

- thorough peer review by experienced researchers in your field

- rapid publication on acceptance

- support for research data, including large and complex data types

- gold Open Access which fosters wider collaboration and increased citations

- maximum visibility for your research: over $100 \mathrm{M}$ website views per year

At $\mathrm{BMC}$, research is always in progress.

Learn more biomedcentral.com/submission 